\title{
Topological optimization of the design of products manufactured by injection molding of plastics
}

\author{
Optymalizacja topologiczna projektowania wyrobów wytwarzanych \\ metodą wtryskiwania tworzyw sztucznych
}

PRZEMYSLAW POSZWA
MAREK SZOSTAK*
DOI: https://doi.org/10.17814/mechanik.2017.11.151

\begin{abstract}
The use of so-called topological optimization for products manufactured by means of injection molding of plastics, is presented. This is a computational method that allows for maximum rigidity of the mold geometry, while reducing its mass. Possibilities of selected numerical tools using topological optimization and constraints in the context of injection molding process, are presented.
\end{abstract}

\section{KEYWORDS: topology optimization, injection molding, plastics}

Development of computational methods, and producing technologies leads to a reduction in weight of components, which translates directly into cost savings and improve economics of the manufacturing process.

Computational methods have evolved with significant increase in computing power of computers. It is possible to more precisely predict the behavior of structural components under the load and thereby reduce their mass (selecting the optimal cross-section). Computational algorithms have been developed which, by modifying the geometry, allow to get the shape of an element to have maximum rigidity and strength [1].

In recent years, there has also been a boom of incremental methods that enable the formation of elements both plastics and metals. The advantage of these methods is the freedom of shapes formation, but the disadvantages are the production time (much longer in comparison with conventional technologies - both deficient and deficientless) and limited dimensions of the elements (although it has been already established technologies that bypass this limitation, including those using a welding to apply successive layers of the workpiece [2]).

Reducing the weight of components is particularly desirable in the aviation industry (in the construction of aircrafts, drones), as it allows to increase the maximum coverage of different solutions. In the case of airplanes, it also allows an increase in payload and in the case of drones - the use of additional functional elements.

Reducing the weight of elements is of great importance also in the automotive industry, as more and more parts are made of plastic. This reduces manufacturing costs and improve vehicle performance.

\section{Topological optimization}

Topology optimization is a numerical method that allows to modify the topology (shape) of the examined element to remove the non-load-bearing areas. In the simplest sense, this problem is referred to as the "material distribution problem". It should be considered that the mechanical element is considered as a body that occupies the $\Omega^{\text {mat }}$ space, being a part of the larger reference space $\Omega$ in the 2$\left(R^{2}\right)$ or 3-dimensional $\left(R^{3}\right)$ space.

The reference domain has been selected for defining the loads and boundary conditions, and it is possible to define a task for optimizing the construction as a problem of finding an optimal choice of $E_{i j k l}(x)$ rigidity tensor, which is variable across the whole domain. If a 2 -linear energy form a (i.e. internal virtual work of the elastic body in the equilibrium situation $u$ for an arbitrary virtual displacement $v$ ) is introduced, also called the compliance criterion, along with the linear form of the strain $\varepsilon$ and the load $l$ :

$$
\begin{gathered}
a(u, v)=\int_{\Omega} E_{i j k l}(x) \varepsilon_{i j}(u) \epsilon_{k l}(v) \mathrm{d} \Omega \\
\varepsilon_{i j}(u)=\frac{1}{2}\left(\frac{\partial u_{i}}{\partial x_{j}}+\frac{\partial u_{j}}{\partial x_{i}}\right) \\
l(u)=\int_{\Omega} f u \mathrm{~d} \Omega+\int_{\Gamma_{\mathrm{T}}} t u \mathrm{~d} \Omega
\end{gathered}
$$

the problem of minimizing deformability (maximum global rigidity) takes the form:

$$
\begin{gathered}
\min _{u \in U, E} l(u) \\
a_{E}(u, v)=l(v) \text { dla każdego } v \in U \\
E \in E_{a d}
\end{gathered}
$$

In other words, it is about obtaining a construction, in which deformation energy at the assumed loads will be as small as possible.

The equilibrium equation is written in a weak, variant form, where $U$ is the kinematically permissible displacement field, $f$ - force applied inside the body, $t$ - force applied at the periphery of domain. Index $E$ indicates that the 2-linear form of energy depends on the design variables, and $E_{a d}$ is the permissible rigidity tensors for a given problem [3].

\footnotetext{
* Mgr inż. Przemysław Poszwa (przemyslaw.b.poszwa@ doctorate.put poznan.pl), dr hab. inż. Marek Szostak (marek.szostak@put.poznan.pl) - Instytut Technologii Materiałów Politechniki Poznańskie
} 
To solve the problem using numerical methods, a typical approach is discretizing of a model using the finite element method. It should be mentioned that in the region of interest, there are two fields: displacement field $u$ and rigidity field $E$. Using the same finite element mesh for the two fields and assuming that $E$ is constant in each element, it is possible to write the discrete form of the problem as:

$$
\begin{gathered}
\min _{u, E_{e}} f^{T} \boldsymbol{u} \\
\boldsymbol{K}\left(E_{e}\right) \boldsymbol{u}=\boldsymbol{f} \\
E \in E_{a d}
\end{gathered}
$$

where: $u$ and $f$ are vectors of displacement and loads, respectively.

The rigidity matrix $K$ depends on the rigidity $E$ in the $e$ numbered element $e=1, \ldots, N$. Then $K$ can be written in the form:

$$
\boldsymbol{K}=\sum_{e=1}^{N} \boldsymbol{K}_{\boldsymbol{e}}\left(E_{e}\right)
$$

where: $K_{e}$ is the rigidity matrix of the element [3].

Topology optimization is usually simplified to a rigidity criterion (e.g. in Autodesk Inventor, Autodesk Fusion 360 [4]). In the case of complex tools, in addition to the rigidity, analysis in terms of minimizing the deformation, stress, temperature optimization, maximizing of the specific frequency and specific values as well as the stability of the structure, can be also performed (Autodesk Nastran [5]).

The obvious advantage of the topology optimization is an effective weight reduction of construction components. In turn, the main drawback is not taking into account the manufacturability of produced elements. In the case of relatively simple shapes, it is possible to perform the milling, but this significantly increases the cost of producing a single piece.

The basic problem with topology optimization for plastic components is the limitations of this production method. Tool in the injection molding process is an injection mold, which at the end of the cycle opens to remove the product. It is the removal of products from the mold that reduces the possibility of forming. Moving parts are used in the injection molds, but they significantly increase the price of such a tool. In the case of optimization of complex geometry, voids not to map in the injection molding process may also occur.

An important problem is the formation of a so-called joining lines. Weld lines are sites, where fronts of the flowing material meet at an angle of less than $135^{\circ}$. At the connection of fronts, the maximum strength is reduced by approximately $20 \%$ (this depends on the connection conditions of the front, type of material, process parameters, etc.) $[6,7]$. This is also the heterogeneity of the material constituting the notch. This type of lines will be present in the most optimized geometries, since they yield the shape of the skeleton after optimization (the more skeletal structure, the more joining lines).

When plastic fronts meet at an angle of more than $135^{\circ}$, the meld lines are formed. These lines, however, to a much lesser extent, cause deterioration of strength properties.

Weakening of the mold in key places can be avoided by controlling the filling profile of the injection mold using the so-called flow promoters and restrictors (corresponding thinning or thickening the walls of the weld) or using a hot runner feed material with controlled opening of the gate.

To compensate the weakening, the RHCM method (Rapid Heat Cycle Molding) can be used, involving the additional heating of the mold before the injection phase. There are several ways of heating the injection mold (resistive, water, steam, infrared, inductive). They differ in capabilities and costs of implementation. The main advantage of the RHCM method is to facilitate the cavity filling with the flowing material, which in contact with the surface of the cavity does not solidify. Due to the plasticized form of material, it does not reach the formation of the bonding resulting in a weakening of the mold. The disadvantages of this method are also the additional costs to be borne at the implementation, and the extended (even several times) cycle time lowering the economics of the process [8].

Due to the problems mentioned in this paper, special use of topological optimization for the design of plastic injection molding products has been proposed. The method consists in optimally designing the ribbing of the mold to increase its rigidity at minimal weight.

\section{Use of topological optimization in articles manufactured by injection molding of plastics}

The object of the analysis was a high density polyethylene HDPE basket. It is used for transportation of, among others, vegetables; during transport, the baskets are stacked in piles. Topology optimization was used to indicate the places where ribbing should be applied.

It was assumed that the basket should have dimensions of $400 \times 300 \times 150 \mathrm{~mm}$. Thickness of the side and bottom walls was assumed for $2 \mathrm{~mm}$. Because of the stacking baskets, restraint of the basket base and the load evenly distributed on the top edge of $1000 \mathrm{~N}$ was also assumed. The analyzes were made in Autodesk Inventor 2017 and Autodesk Fusion 360 software.

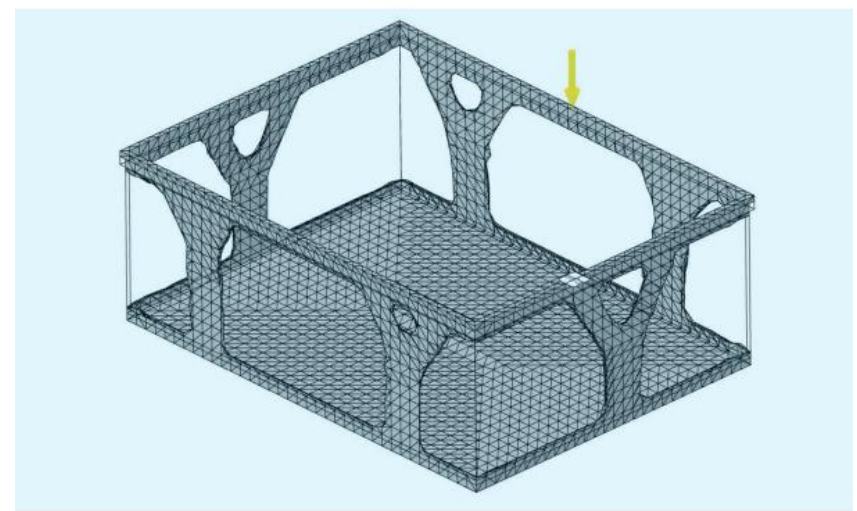

Fig. 1. Results of topology optimization with sparse grid

The first topological optimization studies were performed in Autodesk Inventor. They showed a very strong effect of mesh density and the level of weight reduction of an element on the result obtained. Fig. 1 shows the results $40 \%$ of the material is removed using elements with high side length (average length of the side was approximately $10 \mathrm{~mm}$ ). With the increase in density of the grid, the structure obtained was more fragile, i.e. left areas were much thinner, which is advantageous in the case of molded ribs.

Further calculations were performed for 2 variants of geometry in Autodesk Fusion 360 using a computational cloud. In the first one, it was assumed the target wall thickness for $2 \mathrm{~mm}$, and in the second case, it was thickened to $5 \mathrm{~mm}$ (finally, the ribs had thickness of $1 \mathrm{~mm}$ and height of $3 \mathrm{~mm})$. Two amounts of the removed material were also assumed ( $40 \%$ and $50 \%$ by weight). The average length of the element was approximately $1.25 \mathrm{~mm}$.

Dependence of both the amount of material removed and the thickness of the wall on the achieved results, were observed (figs. 2-5). Increasing the amount of material removed has allowed for much thinner areas requiring 
reinforcement - depending on the thickness, one or more parallel ribs can be used.

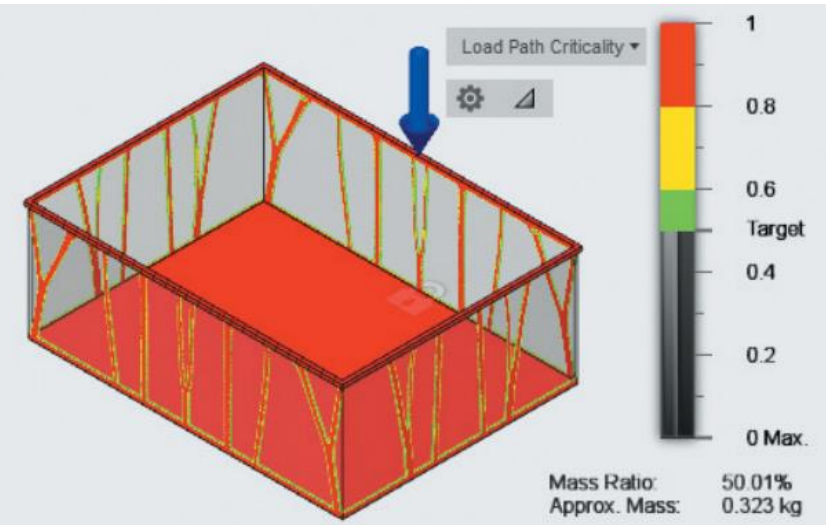

Fig. 2. Result of topological optimization for mass reduction by $50 \%$ assuming a $2 \mathrm{~mm}$ lateral wall thickness

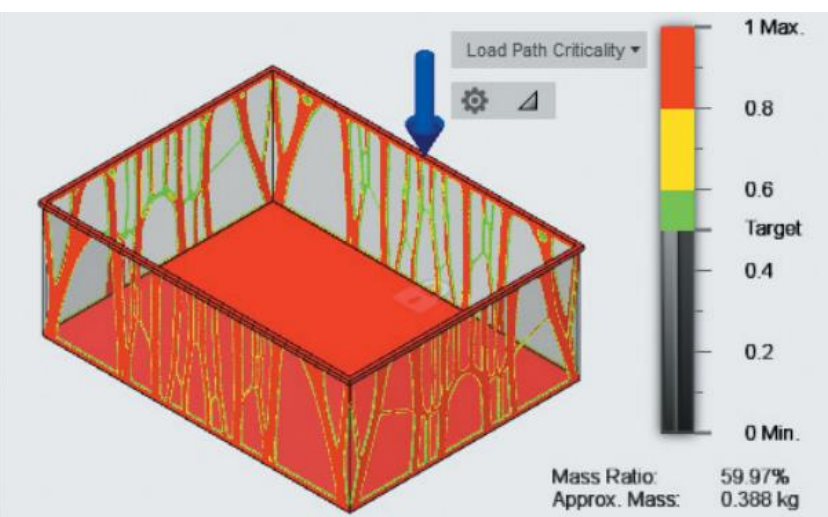

Fig. 3. Result of topological optimization for mass reduction of $40 \%$ assuming a $2 \mathrm{~mm}$ lateral wall thickness

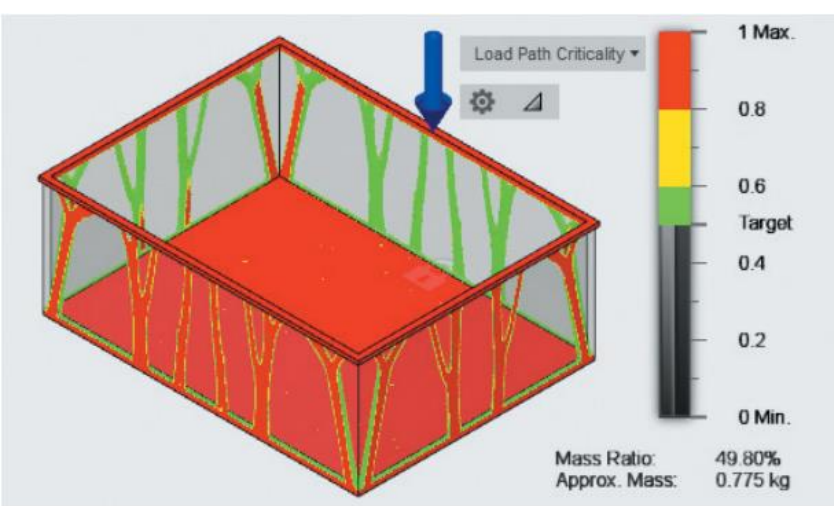

Fig. 4. Result of topological optimization for mass reduction by $50 \%$ assuming the thickness of the sidewall as $5 \mathrm{~mm}$

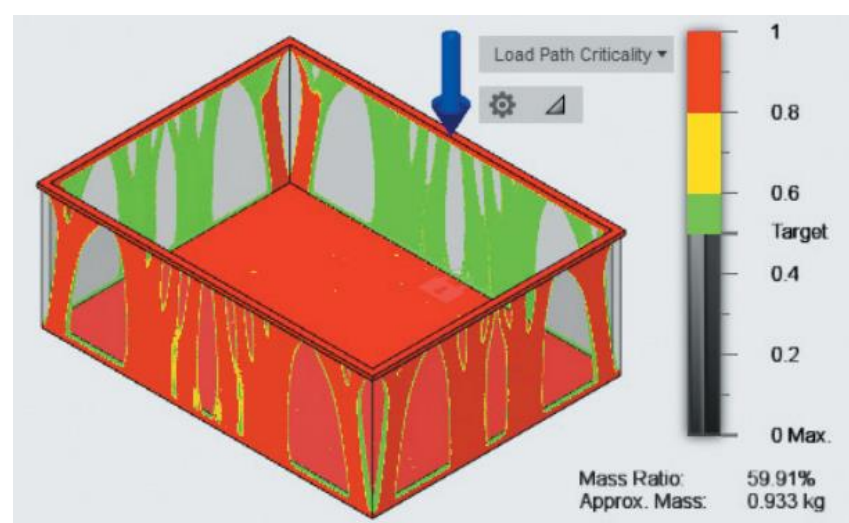

Fig. 5. Result of topological optimization for mass reduction of $40 \%$ assuming the thickness of the sidewall as $5 \mathrm{~mm}$
Increasing the wall thickness from $2 \mathrm{~mm}$ to $5 \mathrm{~mm}$ caused that only at the vertical edges, the algorithm left maximum thickness of "supports" (red) - in other areas, support having a thickness less than the maximum one have been designated (green), as shown in fig. 5. For this reason, to design the ribs, much better solution would be to use thinner walls of the base model.

\section{Conclusions}

Topological optimization can be used to select ribs in articles produced by injection molding of plastics. Depending on the simulation parameters (mesh thickness, amount of material removed), it is possible to obtain areas of different widths, that should be ribbed to improve the rigidity of the structure. For narrow areas, 1 or 2 ribs covering a given region (e.g. fig. 2) should be introduced, and more ribs should be inserted for wider regions (fig. 3-5).

Topological optimization has some drawbacks - it does not take into account the technological aspect of the molding from the view of injection. In addition, the tools used do not allow to affect the topology optimization algorithm, which is prepared for the elements made of metal (relatively small number of very narrow areas removed by so-called filtering [9]). More specialized tools allow the selection of filtering to obtain finely ribbed structures suitable for plastics injection molding products.

\section{REFERENCES}

1. Huang X., Xie Y.M. „Evolutionary Topology Optimization Of Continuum Structures - Methods And Applications". United Kingdom: John Wiley \& Sons 2010.

2. http://mx3d.com/projects/bridge (dostęp: 20.07.2017 r.).

3. Bendsoe M.P., Sigmund O. "Topology Optimization: Theory, Methods and Applications". Berlin Heidelberg: SpringerVerlag 2003

4. Specifications of software: Autodesk Inventor 2017 and Autodesk Fusion 360.

5. Autodesk specification

6. Nastran 2018. 6. Li J., Yang S., Turng L-S., Xie Z., Jiang S. "Comparative Study of Weldline Strength in Conventional Injection Molding and Rapid Heat Cycle Molding". Materiale Plastice. 53, 3 (2016): pp. 448-453.

7. Cloud P.J., McDowell F., Gerakaris G. "Reinforced thermoplastics: understanding weld-line integrity". Plastics Technology. 22, 48 (1976).

8. Mrozek K., Chen S.C. "Selective induction heating to eliminate the fundamental defects of thin-walled moldings used in electrical industry". Journal of Applied Polymer Science. 134, 26 (2017), doi: 10.1002/ app.44992

9. Rozvany G.I.N, Lewiński T. "Topology Optimization in Structural and Continuum Mechanics". Udine: Springer 2014. 